\title{
Spezielle statistische Testverfahren und ihre Anwendung in sozial-psychologischen Untersuchungen
}

\author{
Olga Wälder \\ Technische Universität Dresden
}

\begin{abstract}
Development and/or application of statistical approaches belong to each social-psychological research study. The variety of real problems stimulates also the development of such testing approaches, which are above all free from restrictive assumptions about specified distribution classes. Three empirical tests approaches are proposed in this paper. They are based on some characteristics from spatial statistics and point process theory. Their application for a social-psychological research study is presented.
\end{abstract}

Zusammenfassung: Die Entwicklung und/oder Anwendung statistischer Verfahren gehören zu jeder sozial-psychologischen Studie. Die Vielfalt an realen Fragestellungen regt die Entwicklung auch von solchen Testverfahren an, die insbesondere frei von einschränkenden Annahmen über bestimmte Verteilungsklassen sind. In dieser Arbeit werden drei empirische Testverfahren vorgestellt, die sich auf einige Charakteristiken aus der Räumlichen Statistik und der Punktprozesstheorie stützen. Ihre Anwendung wird anhand einer sozialpsychologischen Studie präsentiert.

Keywords: Räumliche Statistik, Testverfahren, Punktprozess, Simulation.

\section{Einleitung}

Die Entwicklung und/oder Anwendung spezieller statistischer Tests gehören zu jeder sozial-psychologischen Studie, vgl. u.a. O’Brien (1984), Guthrie and Buchwald (1991), Sawilowsky and Blair (1992), Blair and Karniski (1993). Mittlerweile gibt es eine unüberschaubare Anzahl von statistischen Verfahren, die zur Analyse der Ergebnisse solcher Studien eingesetzt werden können. Dennoch erfordert die Vielfalt an realen Fragestellungen immer wieder neue Testverfahren, die vor allem frei von einschränkenden Annahmen über bestimmte Verteilungsklassen sein sollten, wie beispielsweise in Bradley (1968) oder in Wälder (2005).

In Rahmen der Kooperation zwischen den Instituten für Kartographie und für Arbeits-, Organisations- und Sozialpsychologie der Technischen Universität Dresden wurde eine sozial-psychologische Studie durchgeführt, die eine quantitative Analyse der Bildwahrnehmung von Kartenprodukten zum Ziel hatte. Im Verlauf dieser Studie hat sich ein Bedarf an speziellen statistischen Testverfahren aufgezeigt. Die Beschreibung dieser Verfahren und ihre Anwendung sollen den Schwerpunkt dieser Arbeit darstellen. 


\section{Einführung in die Terminologie der räumlichen Stati- stik und der Punktprozesstheorie}

Wir beschränken uns hier nur auf die für die weiteren Untersuchungen relevanten Begriffe und Definitionen. Eine detaillierte Darstellung der Theorie der Punktfelder kann zum Beispiel in Stoyan and Stoyan (1994) und Wälder (1999) gefunden werden. Ein Punktprozess $\Phi$ ist - vereinfacht ausgedrückt - ein mathematisches Modell für die Abhandlungen von Punktmuster, die in einem Raum zufällig verstreut sind.

Das Intensitätsmaß $\Lambda$ spielt in der Theorie der Punktfelder etwa eine ähnliche Rolle wie der Erwartungswert für Zufallsgrößen

$$
\Lambda(B)=\mathrm{E} \Phi(B)
$$

wobei auf der rechten Seite in (1) die mittlere Anzahl der Punkte in einer Borel-Menge $B$ steht. Wenn dieses Intensitätmaß aus (1) eine Dichte $\lambda(x)$ im Sinne von

$$
\Lambda(B)=\int_{B} \lambda(x) d x
$$

besitzt, nennt man diese Intensitätsfunktion Man spricht von homogenen Punktprozessen, wenn gilt

$$
\Lambda(B)=\lambda \nu(B),
$$

wobei mit $\nu(B)$ das Lebesque-Maß bezeichnet wird, d.h. der Flächeninhalt der Menge $B$ im zweidimensionalen Raum.

Als grundlegend in der Punktprozesstheorie gilt das so genannte Campbell-Theorem, das besagt, dass für jede Funktion $f(x)$, die einem Punkt $x$ im Raum eine nichtnegative Zahl zuordnet, die folgenden Relationen bestehen

$$
\mathrm{E} \sum_{x \in \Phi} f(x)=\mathrm{E} \int f(x) \Phi(d x)=\int f(x) \Lambda(d x) .
$$

Nun können Punktprozesse auch markiert werden. Unter einer Markierung von Punktprozessen versteht man eine eindeutige Zuordnung einer Zufallsvariable - der so genannten Marke - zu jedem Punkt des Punkprozesses, vgl. Wälder (1999). Diese Marke kann unabhängig oder abhängig vom Punktprozess verlaufen. Es können auch mehrere Markierungen nacheinander durchgeführt werden. Für markierte Punktprozesse wird der „Arbeitsraum“ um zusätzliche Dimensionen, so genannten Markenachsen, reicher. Die Definition der Intensität eines markierten Punktprozesses $\Psi$ verallgemeinert sich zu

$$
\Lambda_{M}(S)=\mathrm{E} \Psi(S)
$$

vgl. (1), wobei die Menge $S$ eine Teilmenge von $\mathbb{R}^{2} \times M$ für markierte Punktprozesse auf einer Ebene ist. Mit $M$ werden hier die Markenachsen bezeichnet.

Das Cambell-Theorem für markierte Punktprozesse $\Psi$ und eine Funktion $f$, die sowohl von dem Punkt $x$ als auch von einer Marke $m$ abhängt, lautet

$$
\mathrm{E} \sum_{[x, m] \in \Psi} f(x, m)=\mathrm{E} \int f(x, m) \Psi(d(x, m))=\int f(y) \Lambda_{M}(d y) .
$$


Besonders wichtig für die weitere statistische Analyse sind die so genannten Größen zweiter Ordnung aus der Theorie markierter Punktprozesse. Wir werden hier einige davon einführen, um das Verständnis der Terminologie zu erleichtern.

Man bezeichnet das folgende $\mathrm{Ma} \beta \alpha_{f}^{(2)}$ als faktorielles Momentenmaß 2. Ordnung für markierte Punktprozesse

$$
\alpha_{f}^{(2)}\left(B_{1} \times B_{2}\right)=\mathrm{E} \sum_{\left[x_{1}, m_{1} \in \Psi\right]} \sum_{\substack{\left.x_{2}, m_{2} \in \Psi\right] \\ x_{2} \neq x_{1}}} f\left(m_{1}, m_{2}\right) 1_{B_{1}}\left(x_{1}\right) 1_{B_{2}}\left(x_{2}\right)
$$

mit

$$
1_{B}(y)= \begin{cases}1, & y \in B \\ 0, & \text { sonst. }\end{cases}
$$

Falls dieses Maß eine Dichte $\rho_{f}^{(2)}\left(x_{1}, x_{2}\right)$ im Sinne der Relation (4) besitzt, bezeichnet man diese als $f$-Produktdichte

$$
\alpha_{f}^{(2)}\left(B_{1} \times B_{2}\right)=\int_{B_{1}} \int_{B_{2}} \rho_{f}^{(2)}\left(x_{1}, x_{2}\right) d x_{1} d x_{2} .
$$

Nun soll an dieser Stelle eine Deutung von $\rho_{f}^{(2)}\left(x_{1}, x_{2}\right)$ vorgenommen werden. Wir betrachten zwei infinitesimale Kreise mit den Mittelpunkten in (zufälligen!) $x_{1}$ und $x_{2}$ und den Flächen $d F_{1}$ und $d F_{2}$. Hierzu betrachten wir eine Zufallsgröße, die gleich Null ist, wenn nicht in jedem der Kreise genau ein Punkt des markierten Punktfeldes liegt, und die sonst gleich $f\left(m_{1}, m_{2}\right)$ ist. $m_{1}$ und $m_{2}$ bezeichnen die Marken der Punkte in diesen infinitesimalen Kreisen. Der zugehörige Erwartungswert aus (2) und (3) ist näherungsweise gleich $\rho_{f}^{(2)}\left(x_{1}, x_{2}\right) d F_{1} d F_{2}$.

Wir werden weiter isotrope markierte Punktprozesse benutzen, für die gilt

$$
\rho_{f}^{(2)}\left(x_{1}, x_{2}\right)=\rho_{f}^{(2)}(r), \quad r=\left\|x_{2}-x_{1}\right\| .
$$

Dies bedeutet, dass die f-Produktdichte nur vom Abstand zwischen den Punkten und nicht von ihren Positionen im Raum abhängt. Als Markenkorrelationsfunktion in diesem Fall wird der folgende Term bezeichnet

$$
\begin{aligned}
k_{f}(r) & =\frac{\rho_{f}^{(2)}(r)}{\rho_{1}^{(2)}(r)} \frac{\rho_{1}^{(2)}(\infty)}{\rho_{f}^{(2)}(\infty)}=c \frac{\rho_{f}^{(2)}(r)}{\rho_{1}^{(2)}(r)}, \\
c & =\frac{\rho_{1}^{(2)}(\infty)}{\rho_{f}^{(2)}(\infty)}, \quad \rho_{1}^{(2)}(r) \neq 0, \\
\rho_{1}^{(2)}(r) & =\rho_{f}^{(2)}(r) \quad \text { mit } \quad f\left(m_{1}, m_{2}\right)=1 .
\end{aligned}
$$

In Stoyan and Stoyan (1994) werden einige empirische Schätzer der Markenkorrelationsfunktion präsentiert. Wegen der Komplexität ihrer Darstellungen (Quotient zweier erwartungstreuer Schätzer) kann die Erwartungstreue dieser Schätzer meist nicht gewährleistet werden. 
Im Weiteren werden wir den folgenden (Kern-)Schätzer der Markenkorrelationsfunktion benutzen:

$$
\begin{aligned}
\hat{k}_{f}(r) & =c \sum_{\substack{i, j=1, \ldots, n \\
i<j}} f\left(m_{i}, m_{j}\right) w_{i j}(r), \\
w_{i j} & =\frac{G_{i j}(r)}{\sum_{i, j=1, \ldots, n} G_{i j}(r)}, \quad G_{i j}(r) \propto\left|r-\left\|x_{j}-x_{i} \mid\right\| .\right.
\end{aligned}
$$

Die Bezeichnung $\propto$ steht hier für ,,proportional“. Die Funktion $\hat{k}_{f}(r)$ wird weiterhin auch empirische Markenkorrelationsfunktion genannt (siehe Abbildung 1). In Stoyan and Stoyan (1994) wird die folgende Form der Gewichte $G_{i j}(r)$ vorgeschlagen

$$
G_{i j}(r)=\frac{e_{h}\left(r-\| x_{i}-x_{j} \mid\right)}{\nu\left(W_{i} \cap W_{j}\right)}, \quad e_{h}(t)= \begin{cases}\frac{3}{4 h}\left(1-t^{2} / h^{2}\right), & |t| \leq h, \\ 0, & \text { sonst. }\end{cases}
$$

Hier wurde der so genannte Epanechnikov-Kern $e_{h}(t)$ verwendet. Zur Wahl des Fensters $W$ (die unteren Indizes beschreiben seine Translation bis zum entsprechenden Punkt) sowie des Parameters $h$ soll auf Stoyan and Stoyan (1994) verwiesen werden. Meist probiert man verschiedenen Fenster und Parameter solange aus, bis man ,,plausible“ Ergebnisse hat.

Als alternative Kernfunktion kann auch die folgende „robuste“ Funktion benutzt werden

$$
G_{i j}(r)= \begin{cases}1, & r-h \leq\left\|x_{j}-x_{i}\right\| \leq r+h, \\ 0, & \text { sonst. }\end{cases}
$$

Wir gehen zunächst von einem ursprünglichen Punktprozess $\Phi=\left\{x_{i}\right\}$ auf einem Gitter $G$ aus, auf dem die entsprechenden Marken $M\left(x_{i}\right)$ - die Häufigkeit der Blickfixierung verschiedener VPn (Versuchspersonen) im Punkt $x_{i} \in \Phi$ für unsere Anwendung aus Abschnitt 3 - modelliert werden

$$
P(x \in \Phi)=1\{x \in G\} .
$$

Wegen der speziellen Art des markierten Punktprozesses - beispielsweise wegen seiner räumlichen Beschränktheit auf eine Bildebene - kann die Normierungskonstante $c$ aus (4) gleich Eins gesetzt werden.

Es können verschiedene Formen für die Funktion $f\left(m_{1}, m_{2}\right)$ aus (2) verwendet werden, z.B.

$$
\begin{aligned}
& \text { (1) } f\left(m_{1}, m_{2}\right)=m_{1} m_{2}, \\
& \text { (2) } f\left(m_{1}, m_{2}\right)=\left|m_{1}-m_{2}\right| \\
& \text { (3) } f\left(m_{1}, m_{2}\right)=\left(m_{1}\left(\Phi_{1}\right)-m_{1}\left(\Phi_{2}\right)\right)\left(m_{2}\left(\Phi_{1}\right)-m_{2}\left(\Phi_{2}\right)\right), \\
& \text { (4) } f\left(m_{1}, m_{2}\right)=\left|m_{1}\left(\Phi_{1}\right)-m_{1}\left(\Phi_{2}\right)\right|+\left|m_{2}\left(\Phi_{1}\right)-m_{2}\left(\Phi_{2}\right)\right| .
\end{aligned}
$$

Die erste und zweite Funktion aus (7) erlauben eine Aussage bezogen auf denselben Punktprozess, die dritte und vierte Funktion erlauben eine Analyse der absoluten Differenzen zwischen den Marken verschiedener Punktprozesse. 
Bei der weiteren statistischen Analyse wird der Schätzer der unnormierten Markenkorrelationsfunktion stets in der robusten Form verwendet, d.h.

$$
\begin{aligned}
\hat{k}_{f}(r) & =\sum_{\substack{i, j=1, \ldots, n \\
i<j}} f\left(m_{i}, m_{j}\right) w_{i j}(r), \\
w_{i j} & =\frac{G_{i j}(r)}{\sum_{i, j=1, \ldots, n} G_{i j}(r)}, \quad G_{i j}(r)=1\left(r-h \leq\left\|x_{j}-x_{i}\right\| \leq r+h\right) .
\end{aligned}
$$

Die Form (3) der Funktion $f\left(m_{1}, m_{2}\right)$ aus (7) kann zur Konstruktion eines Ähnlichkeitsmaßes $\sigma_{f}$ zwischen Realisierungen von markierten Prozessen wie in (8) sowie zur Clusteranalyse dieser Realisierungen verwendet werden,

$$
\sigma_{f}=\int\left|k_{f}(r)\right| d r
$$

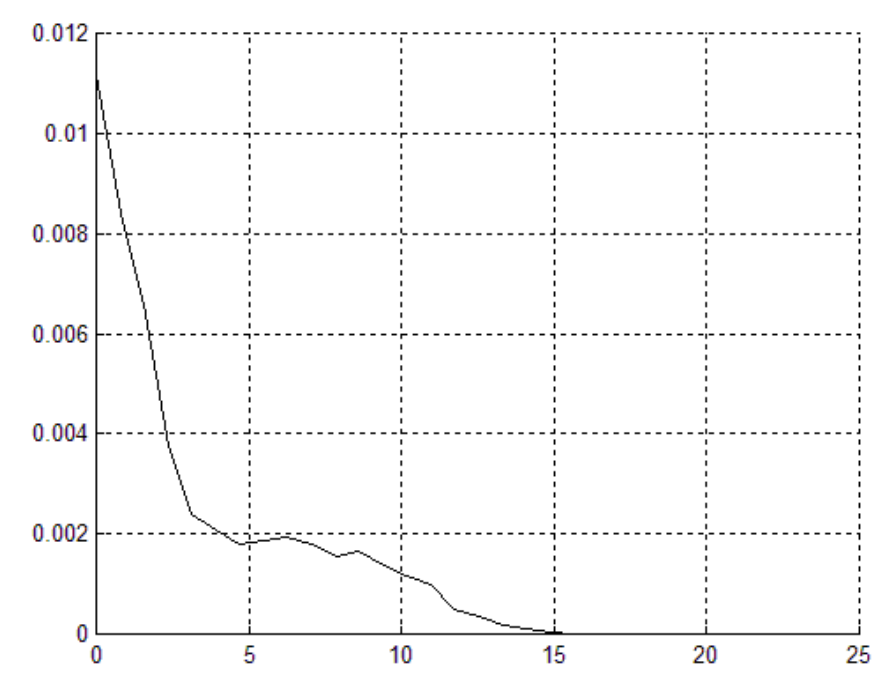

Abbildung 1: Beispiel einer unnormierten $(c=1)$ empirischen Markenkorrelationsfunktion $\hat{k}_{f}(r)$ aus (5) mit $G_{i j}(r)$ aus (6) und $f\left(m_{1}, m_{2}\right)$ der Form (1) aus (7). Die Werte auf der $x$-Achse entsprechen dem Abstand $r$ zwischen den Markenpaaren.

\section{Begriff ,Aufmerksamkeitslandschaft"“ und eine kurze Beschreibung des Experimentes}

Der Begriff „Aufmerksamkeitslandschaft“ stammt aus der Psychologie und wird zur Beschreibung der räumlichen Verteilung der Aufmerksamkeit einer Versuchsperson bei der Betrachtung eines Objektes verwendet. Gewöhnlich tritt ein Bild (oder auch eine Karte) in der Rolle dieses Objektes auf. Diese Aufmerksamkeitslandschaften werden anhand spezieller technischer Mittel erzeugt, die sich sowohl auf Bewegungen als auch auf die 
Fixationsdauer der Augen von Versuchspersonen stützen. Aufmerksamkeitslandschaften werden zur quantitativen Analyse der Bildwahrnehmung herangezogen.

In unseren Untersuchungen wurde ein Ausschnitt einer topographischen Karte zwei Gruppen von jeweils 20 Personen angeboten, siehe Abbildung 2. Anhand dieser Karte sollten sie unabhängig voneinander identische Aufgaben lösen. Während des Lösens dieser Aufgaben wurden jedes Mal Aufmerksamkeitslandschaften erzeugt, siehe Abbildung 3. Diese Aufgaben besaßen verschiedene Schwierigkeitsstufen und wurden so konstruiert, dass die bei einer Lösung erzeugte Aufmerksamkeitslandschaft die weiteren möglichst gering oder gar nicht beeinflussen sollte. Folgende zwei (von insgesamt neun) Aufgaben sollen hier nun näher diskutiert werden:

1. Eine kurze etwa 15 Sekunden lange Betrachtung der Karte im Sinne eines Gemäldes;

2. Einschätzung der stärksten Kartenbelastung (der Begriff „,Kartenbelastung“ ist dabei rein intuitiv zu interpretieren, obwohl es hierfür in der Kartographie eine bestimmte Definition gibt, die sich in erster Linie auf die Objektdichte bezieht).

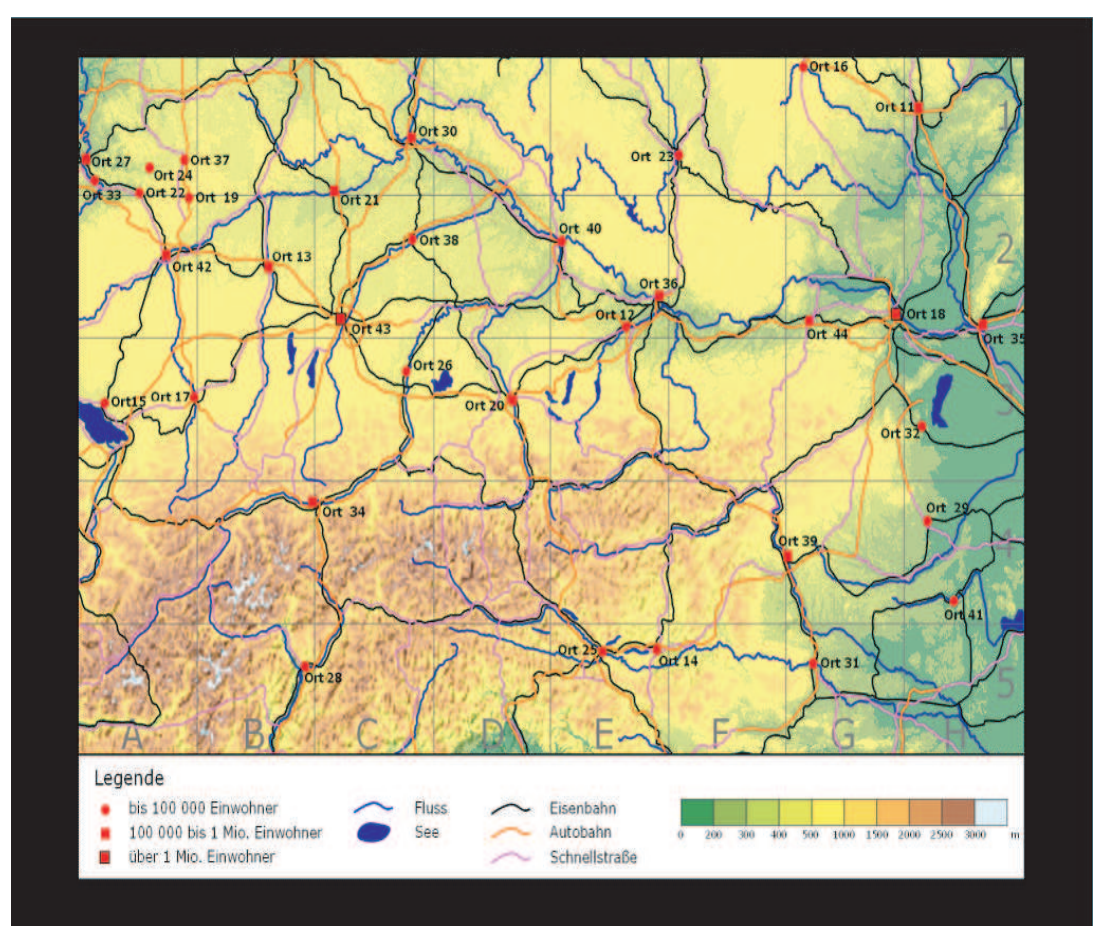

Abbildung 2: Ausschnitt einer topographischen Karte (1008 × 752 Pixel).

Die erste Gruppe (,Experten“) setzte sich aus Personen zusammen, die eine nachweisbare fachliche Kompetenz in der Kartographie besitzen (Studenten der Kartographie, Geographie, Geodäsie usw.). Zu der zweiten Gruppe („Laien“) gehörten Personen, die sich nicht täglich mit der Analyse von Karten befassen (BWL-, Jura-Studenten usw.).

Anhand der Analyse von Aufmerksamkeitslandschaften sollte beispielsweise die Hypothese über den Unterschied in den Betrachtungsprozessen der beiden Gruppen entweder bestätigt oder abgelehnt werden können. 


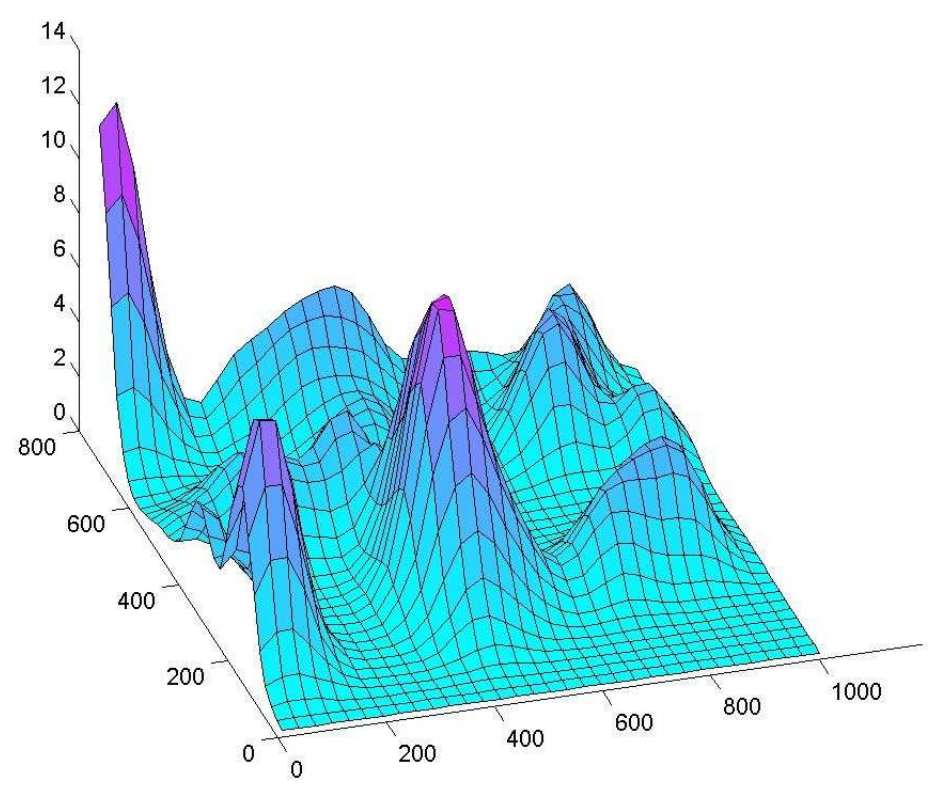

Abbildung 3: Beispiel einer Aufmerksamkeitslandschaft für Aufgabe 1. Die $x$ - und $y$ Achsen entsprechen den Bildkoordinaten in Pixeln. Auf der $z$-Achse ist die Fixationsdauer in Millisekunden dargestellt.

\section{Empirische statistische Testverfahren bezüglich der Identität zweier Betrachtungsprozesse}

Es soll angenommen werden, dass eine Aufmerksamkeitslandschaft eine Realisierung eines zufälligen markierten Punktprozesses ist, der als ein mathematisches Modell des Betrachtungsprozesses $\Psi$ dient. Für jede solche Realisierung kann ein Schätzer der Markenkorrelationsfunktion, d.h. eine empirische Markenkorrelationsfunktion, wie in (5) ermittelt werden. Entscheidet man sich für die Form (1) der Funktion $f\left(m_{1}, m_{2}\right)$ aus (7), so liefert dieser Schätzer immer nicht-negative Werte, weil Aufmerksamkeitslandschaften nicht-negativ sind.

Als erwartungstreuer Schätzer der wahren, aber unbekannten Modell-Markenkorrelationsfunktion kann dann der Mittelwert der empirischen Markenkorrelationsfunktionen für Realisierungen benutzt werden, die zum gleichen Betrachtungsprozess gehören, d.h.

$$
\hat{k}^{(p)}(r)=\frac{1}{N_{p}} \sum_{k=1}^{N_{p}} \hat{k}_{f}^{(p, k)}(r),
$$

wobei mit $N_{p}, p \in\{1,2\}$, die Anzahl von Realisierungen des Prozesses $\Psi_{p}$, und mit $\hat{k}_{f}^{(p, k)}(r)$ die entsprechenden empirischen Markenkorrelationsfunktionen der $k$-ten Realisierung des $p$-ten Prozesses bezeichnet werden. Wir bezeichnen diese Funktionen aus (9) als mittlere empirische Markenkorrelationsfunktionen von Betrachtungsprozessen $\Psi_{1}$ (Experten) und $\Psi_{2}$ (Laien).

Als Streuungsmasse können folgende „Bänder“ (Bereiche zwischen den entsprechen- 


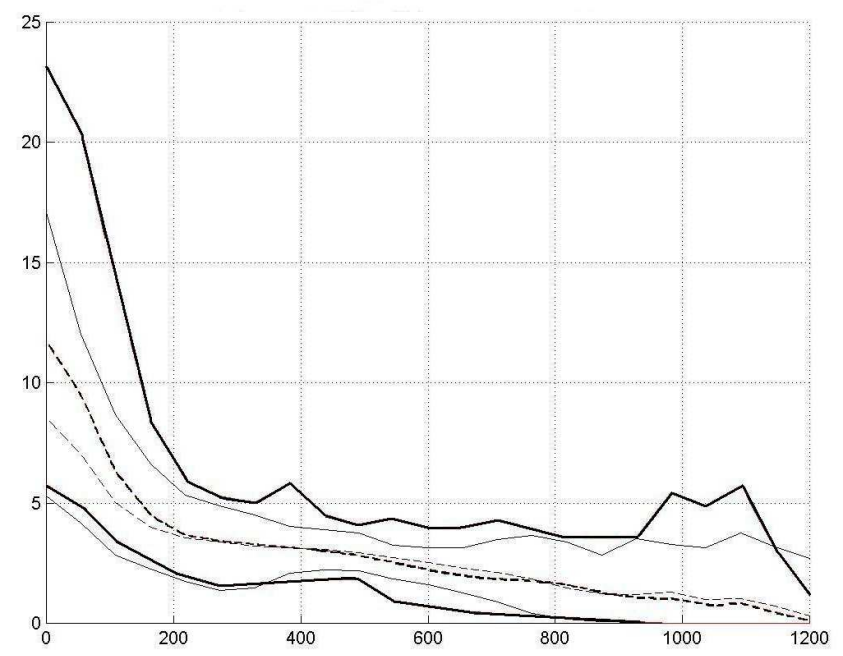

Abbildung 4: Empirische Funktionen $u^{(1)}(r), \hat{k}^{(1)}(r), o^{(1)}(r)$ (dünne Linien, von unten nach oben) und $u^{(2)}(r), \hat{k}^{(2)}(r), o^{(2)}(r)$ (fette Linien, von unten nach oben) für Aufgabe 1. Die Werte auf der $x$-Achse entsprechen dem Abstand $r$ zwischen den Markenpaaren in Bildkoordinaten (Pixeln).

den Ober- und Untergrenzen) für alle $r$ und $p$ benutzt werden (siehe Abbildung 4)

$$
b^{(p)}(r)=\left[o^{(p)}(r), u^{(p)}(r)\right]=\left[\max _{k=1, \ldots, N_{p}}\left[\hat{k}_{f}^{(p, k)}(r)\right], \min _{k=1, \ldots, N_{p}}\left[\hat{k}_{f}^{(p, k)}(r)\right]\right] .
$$

Abbildung 4 zeigt die in (9) und (10) eingeführten Funktionen für das Datenmaterial aus Abschnitt 3 für Aufgabe 1.

Nun soll der empirische Test auf die Identität zweier Betrachtungsprozesse wie folgt formuliert werden:

$H_{0}$ : Die Betrachtungsprozesse sind nicht unterscheidbar, d.h. $\Psi_{1}=\Psi_{2}$.

$H_{1}$ : Die Betrachtungsprozesse sind unterschiedlich, d.h. $\Psi_{1} \neq \Psi_{2}$.

Man berechne die empirische Testgröße

$$
T=2 \frac{\left|b^{(1)} \cap b^{(2)}\right|}{\left|b^{(1)}\right|+\left|b^{(2)}\right|}
$$

wobei mit $|\cdot|$ der Flächeninhalt der entsprechenden Menge bezeichnet wird. Liegt $T$ in einem kritischen Bereich mit $T<1-\alpha, \alpha=0.01 ; 0.05$, so wird die Hypothese $H_{0}$ mit einem Signifikanzniveau $\alpha$ zugunsten der Hypothese $H_{1}$ verworfen. Der (empirische) Fehler 1. Art ( $H_{0}$ verwerfen, obwohl $H_{0}$ richtig ist) beträgt in diesem Fall $\alpha \cdot 100 \%$.

Beispiel 1 (Analyse der Ergebnisse für Aufgabe 1 aus Abschnitt 3): Anhand der Analyse der Ergebnisse aus Abbildung 4 erhält man die Testgröße $T=0.76$. Mit Signifikanzniveau $\alpha=0.05$ muss $H_{0}$ verworfen werden, und die Prozesse werden somit als unterschiedlich angesehen. Somit unterscheidet sich die Betrachtungsart einer Karte bezüglich der beiden Gruppen aus Experten und Laien. 
1. Verallgemeinerung des empirischen statistischen Tests auf die Identität zweier Betrachtungsprozesse

Interpretationen von Ergebnissen hängen meistens von den Modellannahmen ab, die a priori vorausgesetzt werden. Wenn man nun die Modellannahmen weiter verfeinert, kann man die empirischen Markenkorrelationsfunktionen für Realisierungen von Prozessen zusätzlich auf einen ,gemeinsamen Nenner“ bringen, in dem man diese jeweils durch das Quadrat des Integrals der entsprechenden Aufmerksamkeitslandschaft, d.h. des quadrierten Volumens der Landschaft, teilt. Damit kann die „Einheit des Betrachtens“ modelliert werden: Jede VP hat so zusagen eine „Einheit der Aufmerksamkeit“" und verbraucht diese für ein Bild und in einem gewissen Zeitraum auf unterschiedliche Art und Weise. Diese Verallgemeinerung kann zur Erhöhung bzw. Senkung der Variabilität im Band (10) führen.

2. Verallgemeinerung des empirischen statistischen Tests auf die Identität zweier Betrachtungsprozesse

Wenn man die Testgröße (11) durch

$$
T=\frac{\left|b^{(1)} \cap b^{(2)}\right|}{\min \left(\left|b^{(1)}\right|,\left|b^{(2)}\right|\right)}
$$

ersetzt, erhält man einen, die Hypothese $H_{0}$,,begünstigenden“ empirischen Test, mit dem sich auch die absolute Identität einer Menge von Realisierungen und jeder ihrer Untermengen nachweisen lässt.

Fortsetzung des Beispiels 1. In diesem Fall kann $T=0.96$ erreicht werden. Mit Signifikanzniveau $\alpha=0.05$ kann die Hypothese $H_{0}$ nicht verworfen werden, und die Prozesse können nicht als unterschiedlich angesehen werden. Die Betrachtungsart der Experten stellt eher eine Untermenge der Betrachtungsart der Laien dar.

3. Verallgemeinerung des empirischen statistischen Tests auf die Identität zweier Betrachtungsprozesse

Eine empirische Markenkorrelationsfunktion ist invariant bezüglich Spiegelungen oder Drehungen von Realisierungen. Bei einigen Fragenstellungen - wie diese bei der Aufgabe 2 aus Abschnitt 3 - kann es aber wichtig sein, nicht nur distanzbezogene Markenkorrelationen von zwei (oder mehreren) Realisierungen miteinander zu vergleichen, sondern auch die tatsächliche, ,,punktgenaue“ Identität dieser Realisierungen bestätigen bzw. ablehnen zu können. Hierzu kann der folgende Test verwendet werden, vgl. hierzu auch Wälder (2005).

Man untersucht die Markenkorrelationsfunktion der Differenz zweier Prozesse, vgl. Form (3) aus (7). Wenn die Unterschiede unsystematisch, also zufällig sind, dürfen die Ergebnisse dieser Differenz nicht in Clustern auftreten.

Ein absolutes, ,,unkorreliertes“ Chaos kann durch einen korrelationsfreien Gaußschen Prozess modelliert werden. Es werden 500 Simulationen eines solchen Prozesses erzeugt, d.h. für jede Bildmasche wird 500 Mal eine normalverteilte Größe mit dem Mittelwert Null und mit einer Varianz gegeben durch die Varianz der Differenz beider Prozesse generiert (die Varianz einer Reihe von Differenzen für jede Masche). Für jede simulierte Realisierung wird dabei eine empirische Markenkorrelationsfunktion berechnet. 


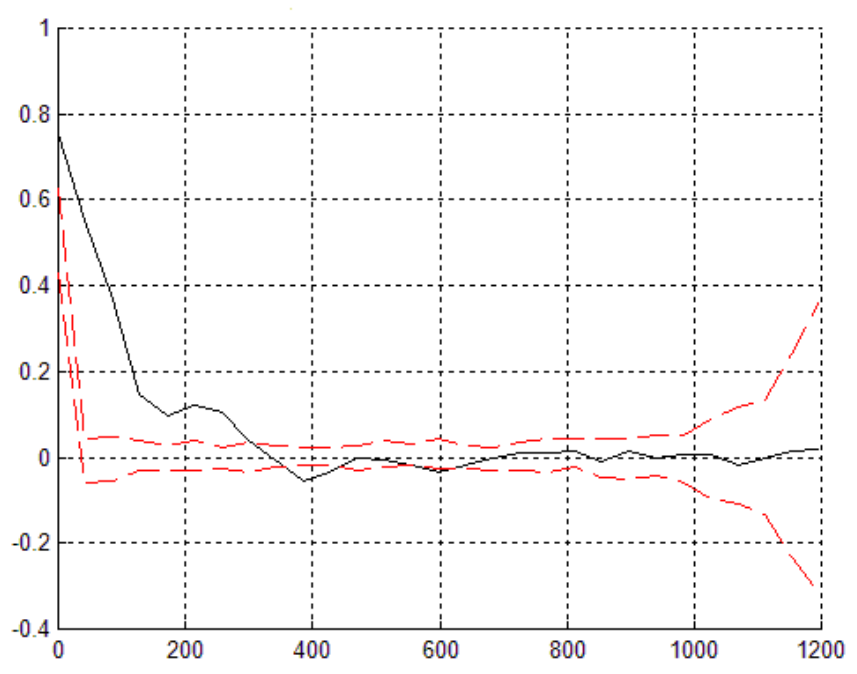

Abbildung 5: Ergebnis eines Tests auf die ,,punktgenaue“ Identität zweier Prozesse: Die simulierte Min/Max-Hülle ist gestrichelt gekennzeichnet. Die durchgezogene Linie zeigt die empirische Markenkorrelationsfunktion der Differenz zweier Prozesse. Die Werte auf der $x$-Achse entsprechen dem Abstand $r$ zwischen den Markenpaaren in Bildkoordinaten (Pixeln).

Anschließend wird dann eine Min/Max-Hülle dieser Markenkorrelationsfunktionen ermittelt, vgl. Wälder (2005). Diese kann der Abbildung 5 entnommen werden. Der Test auf die ,,punktgenaue“ Identität zweier Prozesse lautet dann folgendermaßen:

Liegt nun die ursprünglich berechnete empirische Markenkorrelationsfunktion der Differenz zweier Realisierungen bis zu $M \%, M<(1-\alpha) 100$, in der anhand von Simulationen erzeugten Min/Max-Hülle, so muss die Hypothese $H_{0}$ über die „punktgenaue“ Identität zweier Prozesse mit Signifikanzniveau $\alpha$ zugunsten von $H_{1}$ verworfen werden.

Abbildung 6 zeigt mittlere Aufmerksamkeitslandschaften für die Experten- und Laiengruppe bei der Lösung von Aufgabe 2. Abbildung 7 zeigt die Differenz dieser mittleren Landschaften und eine simulierte Differenz (eine Realisierung des korrelationsfreien Gaußschen Prozesses).

Beispiel 2 (Analyse der Ergebnisse für Aufgabe 2 aus Abschnitt 3): Für Aufgabe 2 wurde der Test auf die ,,punktgenaue“ Identität zweier Prozesse (hier: Unterschiede in der Interpretation des Begriffes ,,Kartenbelastung“ bei Experten und Laien) benutzt, siehe hierzu die oben diskutierte 3. Verallgemeinerung.

Der prozentuelle Anteil der durchgezogenen Linie in der Min/Max-Hülle in Abbildung 7 entspricht 62\%. Mit Signifikanzniveau $\alpha=0.05$ muss die Hypothese $H_{0}$ verworfen werden und somit die ,punktgenaue“ Interpretation der "Kartenbelastung” bei Experten und Laien doch als unterschiedlich angesehen werden. 

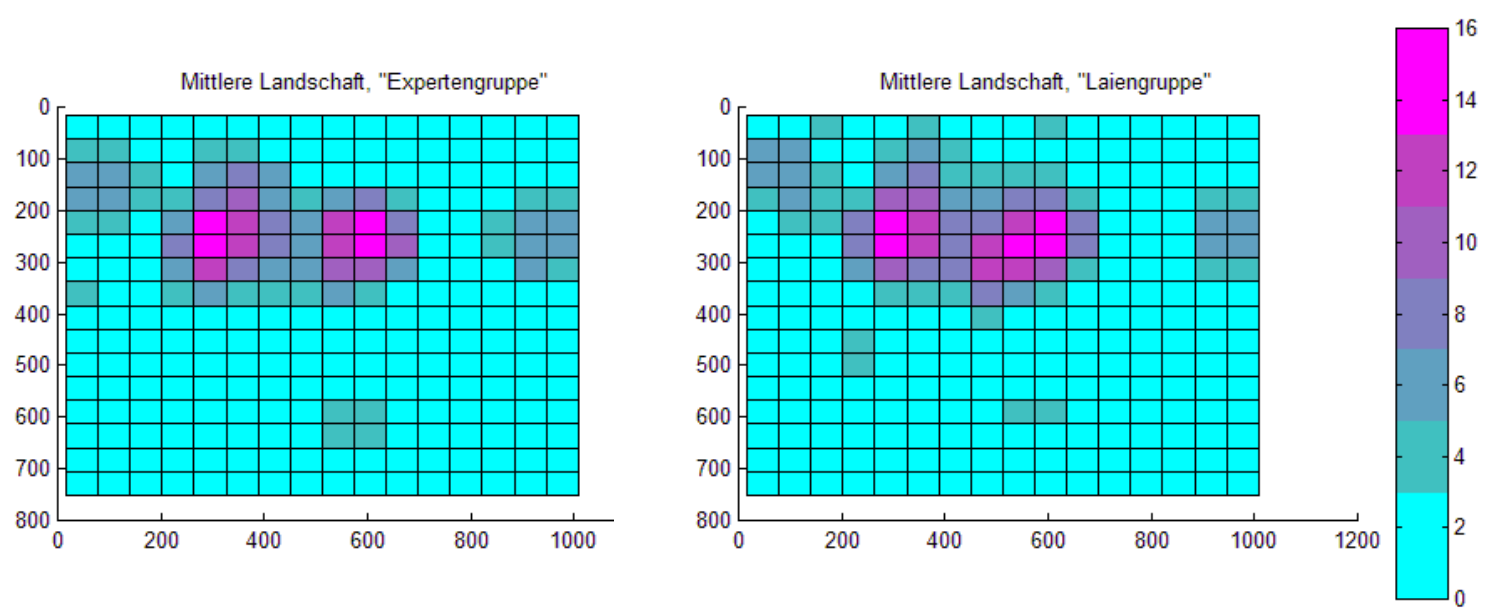

Abbildung 6: Mittlere Aufmerksamkeitslandschaften für die Experten- und Laiengruppe bei der Lösung von Aufgabe 2.
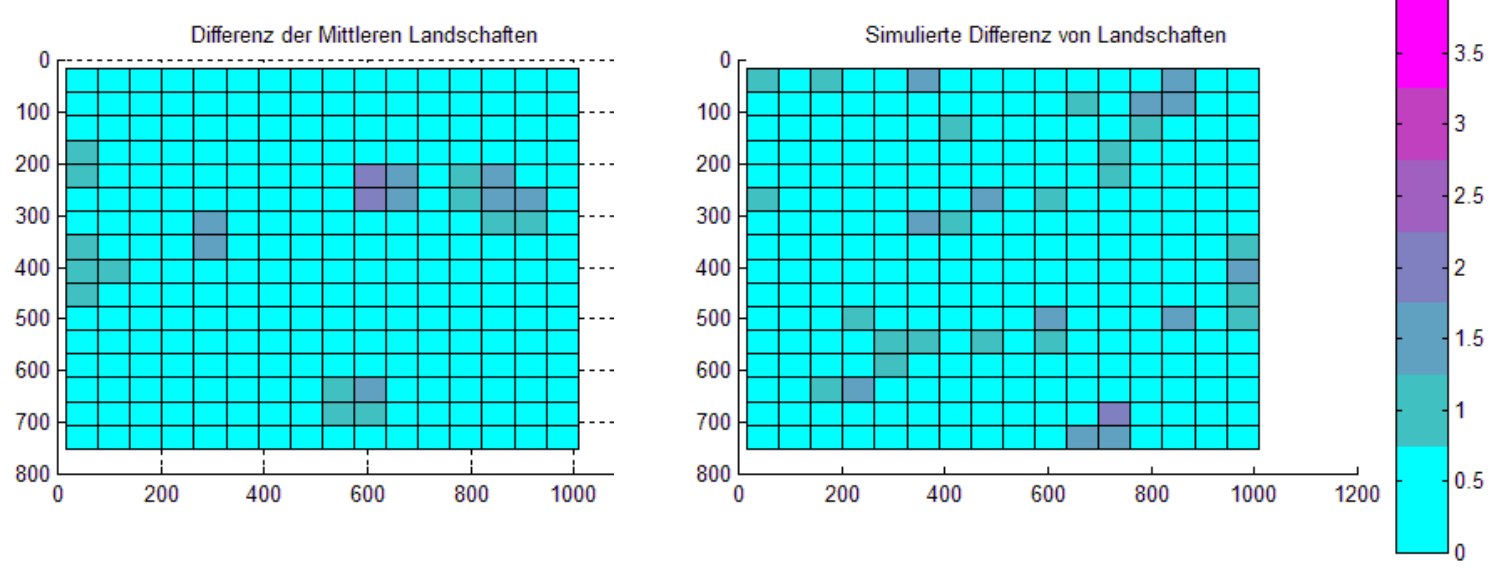

Abbildung 7: Differenz der mittleren Landschaften und eine simulierte Differenz.

\section{Ausblick und Diskussion}

Mithilfe von Verfahren der räumlichen Statistik und der Punktprozesstheorie lassen sich einige nützliche empirische Tests konstruieren. Diese Tests wurden nun nicht ausschließlich für sozial-psychologische Untersuchungen entwickelt, sondern stellen eine sinnvolle Ergänzung für herkömmliche multidimensionale statistische Verfahren dar. Sie sind besonders für den Fall geeignet, dass gewisse Testannahmen bei „klassischen Tests“ anhand des Datenmaterials nicht gewährleistet werden können.

\section{Literatur}

Blair, R. C., und Karniski, W. (1993). An alternative method for significance testing of waveform difference potential. Psychophysiology, 30, 518-524. 
Bradley, J. V. (1968). Distribution-free statistical tests. Englewood Cliffs, NJ: PrenticeHall.

Guthrie, D., und Buchwald, J. S. (1991). Significance testing of difference potentials. Psychophysiology, 28, 240-244.

O'Brien, P. C. (1984). Procedures for comparing samples with multiple endpoints. Biometrics, 40, 1079-1087.

Sawilowsky, S. S., und Blair, R. C. (1992). A more realistic look at the robustness and type II error properties of the t-test to departures from population normality. Psychological Bulletin, 111, 352-360.

Stoyan, D., und Stoyan, H. (1994). Fractals, Random Shapes and Point Fields. Chichester: John Wiley.

Wälder, O. (1999). Markierungen und Verdünnungen von Punktprozessen. Unpublished doctoral dissertation, Fakultät für Mathematik und Informatik, TU Bergakademie Freiberg.

Wälder, O. (2005). Ein empirisches statistisches verfahren zur indikation von datenabnormitäten mittels einer speziellen analytischen darstellung des georeliefs. Austrian Journal of Statistics, 34, 251-261.

Adresse der Autorin:

Olga Wälder

Institut für Kartographie

Technische Universität Dresden

Mommsenstrasse 13

D-01062 Dresden

Deutschland

Tel. +49 351 463/36200

Fax +49 351 463/37028

E-Mail: Olga.Waelder@tu-dresden.de

http://web.tu-dresden.de/kartographie 